\title{
Technology fusion of metal injection moulding and selective laser melting for the manufacture of complex and multifunctional (hydraulic) components
}

\author{
Martin Herold ${ }^{1)}$, Camilo Zopp ${ }^{1)}$, Oliver Neiske ${ }^{2)}$, Frank Schubert ${ }^{1)}$, Jan Hustert ${ }^{2)}$, Wolfgang Nendel ${ }^{1)}$, \\ Lothar Kroll') \\ 1) Department of Lightweight Structures and Polymer Technology, martin.herold@mb.tu- \\ chemnitz.de, camilo.zopp@mb.tu-chemnitz.de,frschu@hrz.tu-chemnitz.de, \\ nendel.wolfgang@mb.tu-chemnitz.de, lothar.kroll@mb.tu-chemnitz.de, Chemnitz University of \\ Technology, Reichenhainer Straße 31/33, 09126 Chemnitz, Germany \\ 2) Parker Hannifin Manufacturing Germany GmbH \& Co. KG, oneiske@parker.com, Am Metallwerk \\ 9, 33659 Bielefeld, Germany
}

\section{Keywords}

Additive Manufacturing, Metal Injection Moulding, Selective Laser Melting, Fitting, Joint Strength

\begin{abstract}
Selective laser melting (SLM) and metallic injection moulding (MIM) are established processes for the production of high-performance metallic components for small and large series. In the aerospace industry, where very high demands are placed on materials and components, both processes are still considered to be relatively new. In both processes, the conventional titanium alloy Ti-6Al-4V can be used in the form of powder. Currently, both technologies are only considered separately. By fusing components of the same type, multifunctional components with a high lightweight construction potential can be produced.

In order to generate direct material fusion, the MIM component must be mechanically processed accordingly. In addition, suitable SLM process parameters must be developed in order to ensure both generative construction and high joint strength. To this end, a characterisation of the joining zone and the static joint strength was carried out. Furthermore, pressure test samples were designed and examined both statically and for fatigue strength. Thus, a high static joint strength could be proven. The compression test samples also withstood a fatigue strength of over 1 million cycles.
\end{abstract}

\section{Introduction}

The production of cost-effective metallic system components, especially for aerospace applications, is to be achieved by future hybrid manufacturing processes. In this context, the key technologies of selective laser melting (SLM) and metal injection moulding (MIM) have emerged. Selective laser melting is characterised by the production of near-net-shape and geometrically complex components with high functional density. For the production of such components, a CAD model is required, which is broken down into horizontal layers of a defined thickness in a slicing process. Following this, the layer-by-layer additive build-up on a metallic substrate can take place through targeted laser exposure.

The MIM process is ideally suited for both high-volume and small series production with moderate geometric complexity. While the additive manufacturing process only requires metallic powder as base material, the metallic injection moulding process requires an additional feedstock (binding agent). After the injection moulding process, the "green part" is debound and sintered to obtain the finished part.

The use of titanium alloys, such as Ti-6Al-4V, is known in both technologies from the state of the art [15]. It is characterised by e.g. high specific strength, corrosion resistance, low density and good weldability. A hybridisation of an additively manufactured Ti-6Al-4V structure and a substrate plate of the same type was shown in [6-8]. By using individual components from the MIM and SLM processes 
with reduced geometry and weight, it is possible to achieve weight savings in the overall system, which in the aircraft construction industry, for example, leads to savings in fuel consumption and thus makes a significant contribution to environmentally friendly air traffic [9].

Within the framework of the investigations, metallic subcomponents resulting from the MIM process are to be made available for direct material fusion. The finalisation of the novel fitting concept was achieved by functionalisation in the SLM process. Such a fusion of metallic subcomponents and their characterisation is not known from the current state of the art. This combination of technologies means that economic manufacturing and assembly processes for the production of modular, hybrid lightweight components can be derived and ensured.

\section{Materials and methods}

\subsection{Materials and manufacturing process}

The powder material used for the SLM process was a conventional titanium alloy Ti-6Al-4V (grade 23) with a standard particle size of 20-63 $\mu \mathrm{m}$. The titanium powder was produced by plasma atomisation and exhibits a spherical shape. After the atomisation process no pores are visible in the particle. The particle size distribution is given in Table 1.

Table 1 Particle size distribution of the used SLM powder

\begin{tabular}{lc}
\hline Particle size distribution & Ti-6Al-4V \\
\hline$D_{10}[\mu \mathrm{m}]$ & 23 \\
$D_{50}[\mu \mathrm{m}]$ & 41 \\
$D_{90}[\mu \mathrm{m}]$ & 61
\end{tabular}

The additive build-up was carried out on the SLM $250 \mathrm{HL}$ laser beam melting system from SLM Solutions $\mathrm{GmbH}$. A fibre laser with a maximum output of $400 \mathrm{~W}$ was used as energy source. The samples and individual seams were built up at a constant layer thickness of $30 \mu \mathrm{m}$ under an argon atmosphere with an oxygen content of $<0.05 \%$. As scanning strategy, a stripe exposure in $X / Y$ direction with a vector length of $10 \mathrm{~mm}$ was chosen. The increment angle was $79^{\circ}$. A process window was identified, resulting in structures with a density of almost $100 \%$.

For a tight and strong joint, the pre-treatment condition of the substrate is decisive, for this reason, a surface pre-treatment is indispensable. Sandblasting was therefore chosen as the pre-treatment method for the MIM subcomponent. As blasting medium, irregular white corundum particles (particle size: 125$180 \mu \mathrm{m}$ ) were chosen. Using this process, homogeneous and reproducible surfaces could be achieved. For the additive build-up of the tensile and compression test samples, additional modifications of the substrate plate were necessary in each case.

The titanium alloy Ti-6AI-4V also served as base material for the MIM process. Within the context of the investigations, a gas-atomised spherical powder with a particle size of D90 $(25 \mu \mathrm{m})$ was used for relatively large components. As such, this is somewhat finer than the powder used for the SLM process. For the MIM sub-component, various material batches (PM 300, PM 400, PM 600) were also examined for their basic processability. The components were injected on a Krauss Maffei 80-380CX hydraulic injection moulding machine with a special MIM screw. Sintering was carried out in the Elnik 3045 T50XHB furnace under argon inert gas atmosphere. The green part weight was approx. $145 \mathrm{~g}$, the injection pressure was $1600 \mathrm{bar}$, melting temperature $185^{\circ} \mathrm{C}$ and mould temperature $60^{\circ} \mathrm{C}$. Sintering was carried out at a temperature of $1,350^{\circ} \mathrm{C}$ with $1 \mathrm{~h}$ holding time. In order to limit the warpage caused by the sintering process, shrinkable substrates of the feedstock were used, which were prepared with the help of a ceramic spray coating.

For the MIM-SLM joining, a test sample was produced with different pre-treatment conditions. This resulted in mean roughness depths $\left(R_{z}\right)$ of 6.93 to $27.57 \mu \mathrm{m}$. Preliminary tests proved that a minimum surface roughness of approx. $20 \mu \mathrm{m}$ is required for a precise powder deposition. In order to achieve the required roughness, however, suitable mating surfaces had to be prepared using a milling process and 
subsequent surface treatment. For this purpose, the samples were first sandblasted with coarse white corundum (particle size: $425-600 \mu \mathrm{m}$ ) or fine white corundum (particle size: $125-180 \mu \mathrm{m}$ ) for one minute.

\subsection{Joining zone}

In order to investigate the influence of the process parameters on the joining zone, individual seams (weld beads) were first generated on the MIM samples, which were previously sandblasted. The process parameters such as laser power (175 and $200 \mathrm{~W})$, scanning speed $(700,900$ and $1100 \mathrm{~mm} / \mathrm{s})$ and laser focus $(70$ and $80 \mu \mathrm{m})$ as well as the number of initial exposures (1 and 3 ) were varied.

\subsection{Joint strength}

The joint strength between the MIM and SLM subcomponents was determined in accordance with DIN EN ISO 6892-1. The shape of the tensile test sample (B5 $\times 25)$ was based on DIN 50125. For this purpose, first MIM solid cylinders ( $\mathrm{h}: 8 \mathrm{~mm}, \varnothing: 10 \mathrm{~mm}$ ) were prepared, inserted into the substrate plate and an SLM solid cylinder (h: $26 \mathrm{~mm}, \varnothing: 10 \mathrm{~mm}$ ) was added. The cylinder was then turned and the other side of the MIM subcomponent was pressed on accordingly. The schematic structure can be seen in Figure 1. The sample was then mechanically post-treated in accordance with DIN EN ISO 6892-1. Three samples were tested both in "as built" and "as built + HIP" condition. The HIP process or hot isostatic pressing is used to reduce the pore content resulting from the additive manufacturing process. The tensile test was performed on the Zwick/Roell Z100. The test speed was $0.00671 / \mathrm{s}$.

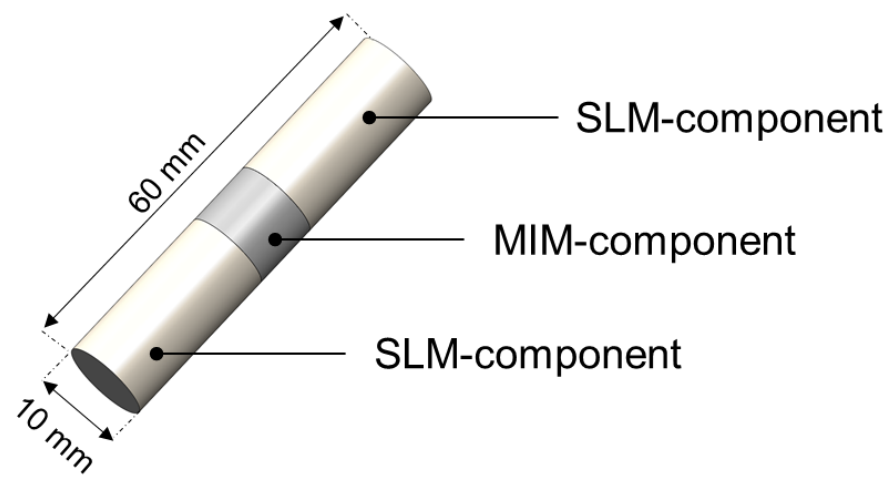

Figure 1: Structure of the tensile test sample made of the respective technology components

\subsection{Hydrostatic testing}

In order to characterise the static and dynamic joint strength or leak tightness under internal pressure load, MIM/ALM hydrostatic test samples were constructed as shown in Figure 2. Due to the production tolerances, deviations of approx. $0.1 \mathrm{~mm}$ and up to $0.5^{\circ}$ occur in the additive structure. As a result, wall thickness ratios must be adjusted so that a maximum stress of approx. $300 \mathrm{MPa}$ is achieved in the idealised component. To reduce notch effects, especially at the contact pressure point, the test samples were mechanically post-treated on the inside. The static pressure test was carried out on a Maximator pressure test stand with a Uraca HP 32-8 test pump. A water/glycol mixture served as pressure medium. A test pressure of 550 bar and a holding time of 5 min were specified.

Five samples each were mounted on the FLS500/5000 high-pressure pulse test stand for the dynamic test. The pressure rise rate was $5000 \mathrm{bar} / \mathrm{s}$, the test pressure $450 \mathrm{bar}$ and the frequency $3 \mathrm{~Hz}$. 


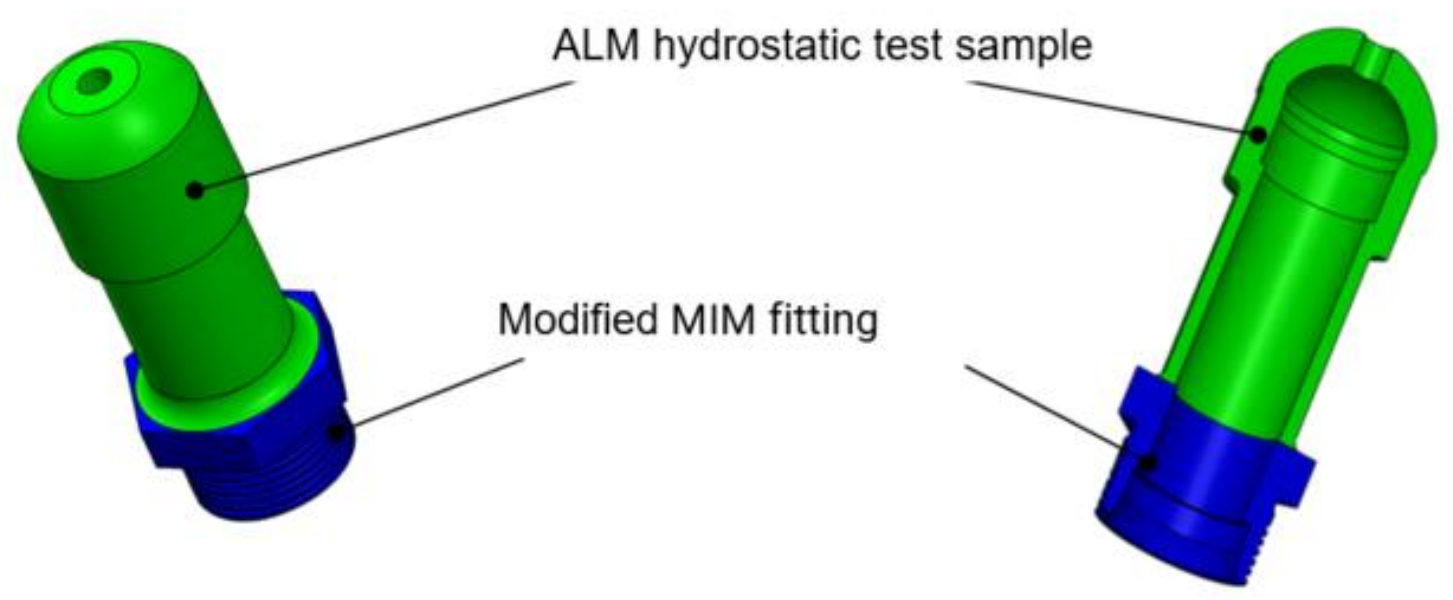

Figure 2: Structure of the hydrostatic test samples and sectional view of the respective technology components

\section{Results}

\subsection{MIM subcomponent}

The density of the material batches for PM300 + PM400 was $98.3 \%$, the tensile strength was $920 \mathrm{MPa}$. The elongation at break is more than $10 \%$, which also corresponds to the target value for MIM parts made of Ti-6Al-4V. A reduced tensile strength of $900 \mathrm{MPa}$ was determined for the samples from the PM600 batch at the same density values. The elongation at break in the component varied between 3 $\%$ and $10 \%$. This can be explained by injection moulding errors, which mainly occurred due to geometry.

\subsection{Joining zone}

A characterisation of the joining zone was carried out according to the process parameters and initial exposure. As the first layer is deposited manually, fluctuations in layer thickness have to be considered. In the first step, a single initial exposure was implemented. This showed a near-surface formation of the joining zone. By changing the single to a triple initial exposure strategy, however, a pronounced root penetration could be demonstrated. For this reason, the further investigation was carried out exclusively with a triple initial exposure.

It is apparent that a higher laser power increases the root penetration depth. Furthermore, the laser focus has a considerable influence on the joining zone. The best possible joining zone could be achieved with a comparatively small laser focus (cf. Figure 3). 


$$
\begin{aligned}
& P=175 \mathrm{~W} \\
& \mathrm{v}=700 \mathrm{~mm} / \mathrm{s} \\
& \mathrm{F}=70 \mu \mathrm{m}
\end{aligned}
$$

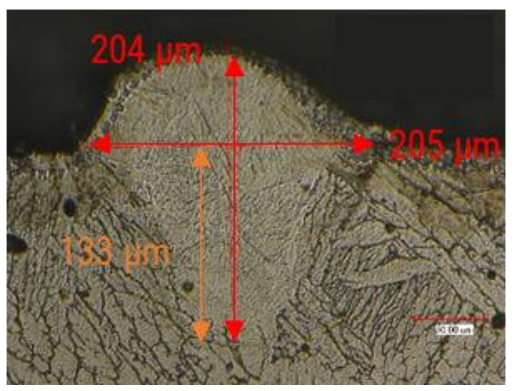

$$
\begin{aligned}
& P=175 \mathrm{~W} \\
& \mathrm{v}=700 \mathrm{~mm} / \mathrm{s} \\
& \mathrm{F}=80 \mu \mathrm{m}
\end{aligned}
$$

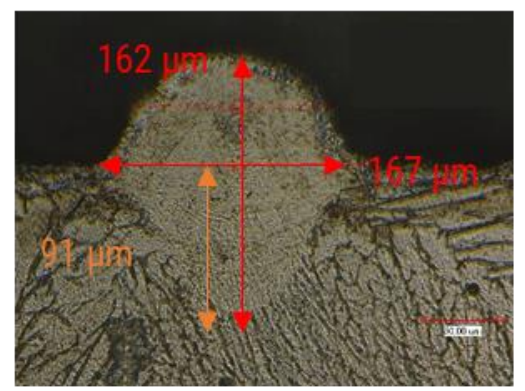

$$
\begin{aligned}
& P=200 \mathrm{~W} \\
& \mathrm{v}=700 \mathrm{~mm} / \mathrm{s} \\
& F=80 \mu \mathrm{m}
\end{aligned}
$$

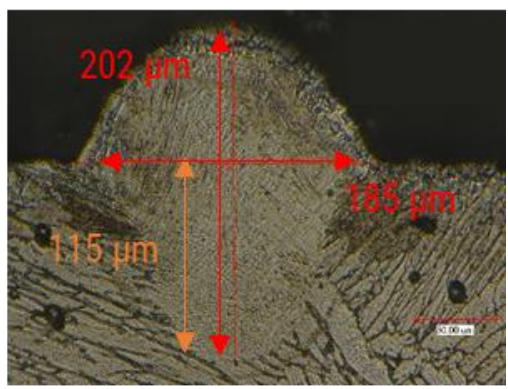

Figure 3: Formation of the joining zones depending on the selected process parameters

Furthermore, conclusions could be drawn about the joining zone from the defined surface roughness. It was found that the different surface morphology leads to changes in energy absorption behaviour. The surface blasted with coarse corundum produced a higher root penetration than the surface treated with the fine white corundum (cf. figure 4). This is due to the greater surface roughness, which amounts to approx. $32 \mu \mathrm{m}$ after the mechanical surface treatment and is thus more than twice as deep as with fine white corundum.

$$
\begin{aligned}
& \mathrm{P}=225 \mathrm{~W} \\
& \mathrm{~V}=900 \mathrm{~mm} / \mathrm{s} \\
& \mathrm{F}=70 \mu \mathrm{m}
\end{aligned}
$$

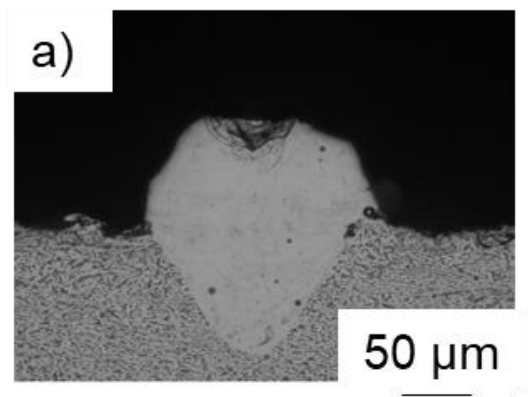

$$
\begin{aligned}
& \mathrm{P}=225 \mathrm{~W} \\
& \mathrm{v}=900 \mathrm{~mm} / \mathrm{s} \\
& \mathrm{F}=70 \mu \mathrm{m}
\end{aligned}
$$

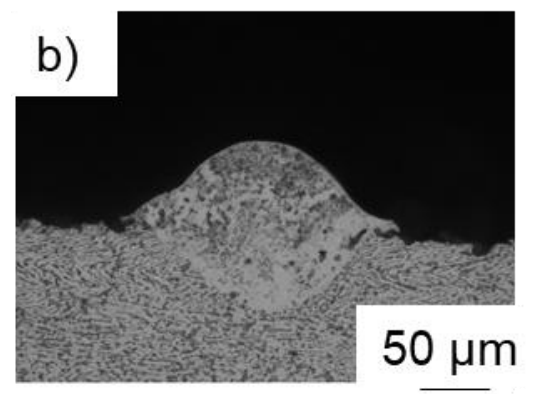

Figure 4: Root penetration depending on the blasting material a) coarse corundum and b) fine corundum

It also became apparent that the SLM parameters with a comparatively good root penetration were not suitable for the generative construction of functional elements. This is due to the fact that this high root penetration melts a comparatively large area, but also induces a high degree of residual stress in higher layers by re-melting the material. These residual stresses lead to considerable warpage at greater component heights. In this context, significantly reduced surface qualities as well as geometric deviations could be detected. For this reason, a hybrid data set was developed for the further development of the SLM subcomponent.

\subsection{Joint strength}

Within the scope of the investigation, a Young's modulus of approx. $115 \mathrm{GPa}$, a tensile strength of approx. $934 \mathrm{MPa}$ and an elongation at break (A25) of approx. $3.5 \%$ could be determined for the "as built " condition. The samples failed only in the area of the MIM cylinder. Figure 5 shows the tested samples after fracture. According to this, there is a higher bond strength between the MIM/SLM interface. Typical Young's modulus and strength values were achieved for this titanium alloy. 


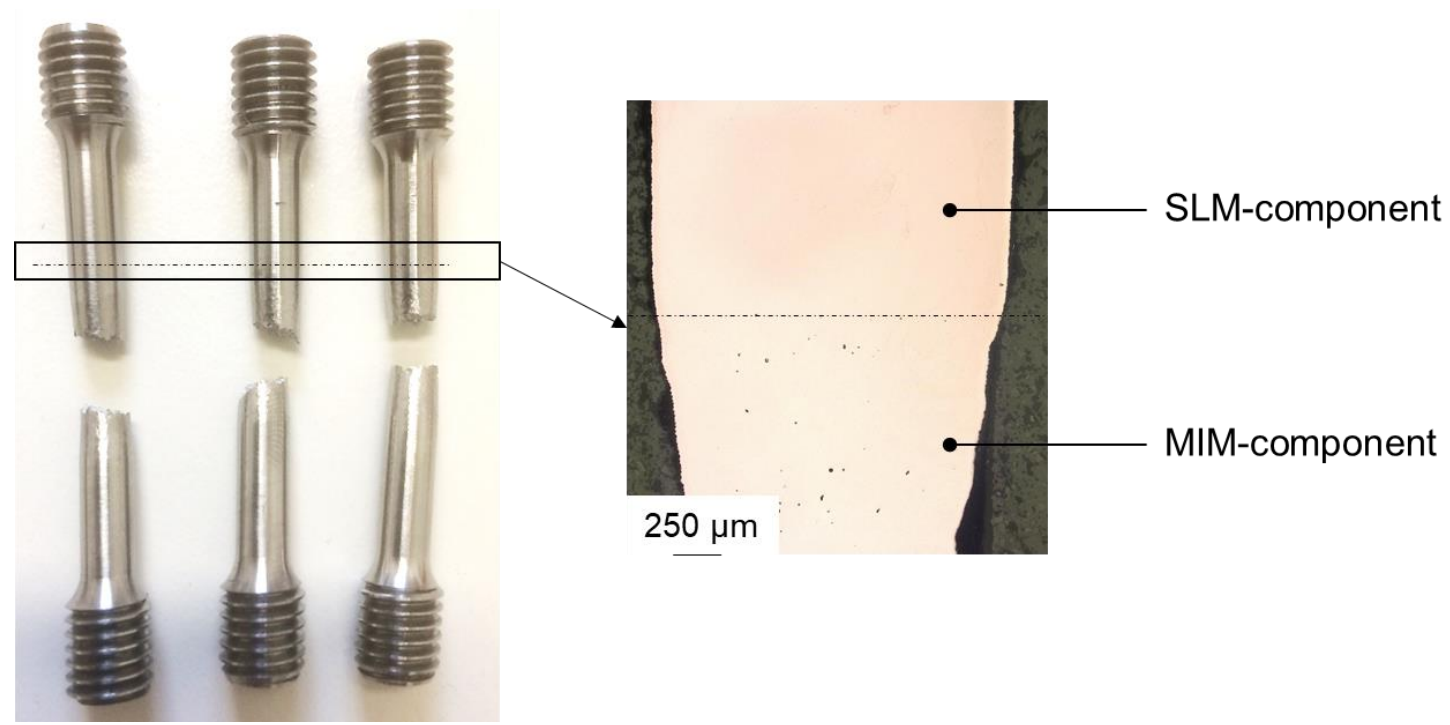

Figure 5: Fracture failure after the quasi-static tensile test and MIM-SLM interface (dashed line)

Furthermore, it is evident that no significant interface (joining zone) is formed between the MIM and SLM components. However, pores are visible in the MIM component, while the SLM component has a material density of almost $100 \%$ (cf. Figure 6). After the HIP process, the Young's modulus could be increased to approx. $119 \mathrm{GPa}$. The tensile strength also increased to approx. 1,017 MPa. The elongation at break (A25) was about $6.0 \%$. However, the latter showed a relatively high scattering (approx. $\pm 2 \%$ ). Analogous to the "as built" condition, the samples only failed in the MIM cylinder. The transition area is comparable to the "as built" condition.

\subsection{Hydrostatic testing}

Figure 7 shows the hydrostatic test samples for the pressure pulse test and the design for a stressed component at expected burst pressure. During the static tests up to 550 bar, no pressure drop was detected. According to this, there was no failure at the interface between the MIM and SLM subcomponents.
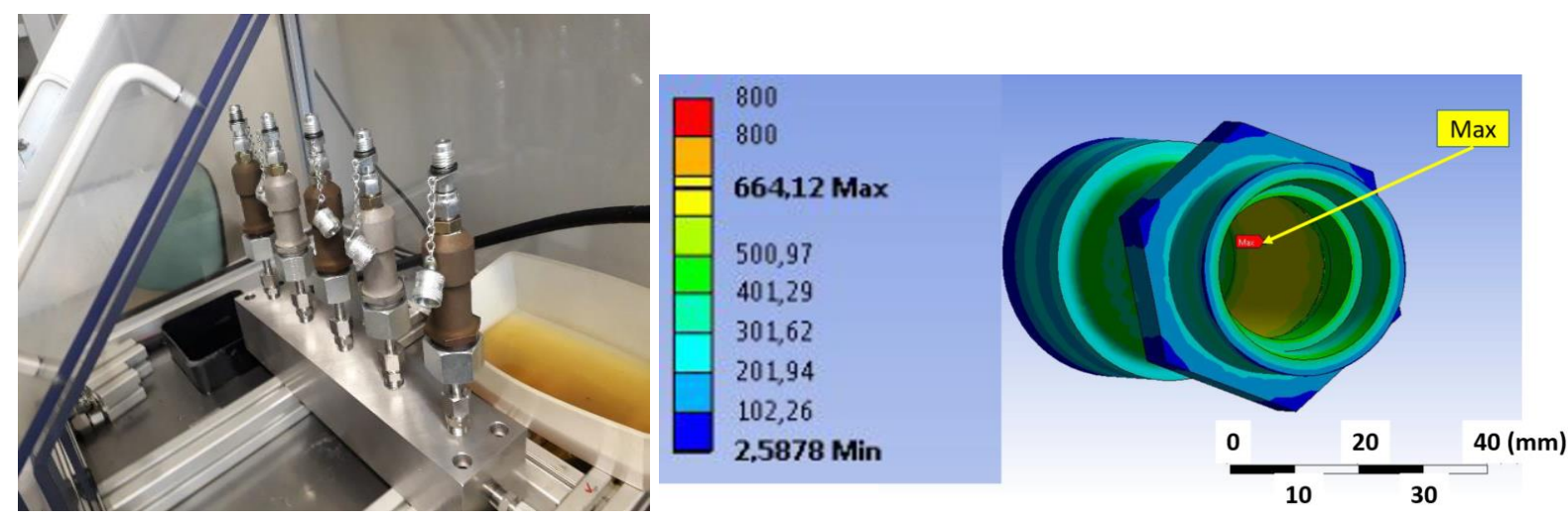

Figure 6: Constant pressure test (left) and simulated design (right) for component stress

The pressure impulse test (5000 bar/s) was completed without failure at the interface after 1 million cycles per test, whereby the fatigue strength could be demonstrated both for "as built" and for thermally post-treated samples (vacuum annealed, inert gas annealed or "HIP'd") up to at least approx. $300 \mathrm{MPa}$. The "as-built" samples, however, showed settling effects in the screw areas around the threads, making retightening beyond the permissible torque necessary to ensure tightness. 


\section{Discussion}

During the investigations, a high joint strength was found between the MIM and SLM subcomponents. In addition to the similar material composition, this is also due to the comparatively high root penetration of the individual SLM seams. The MIM component was tempered in the same way as the substrate plate of the SLM process, which counteracts the thermal residual stresses. However, additional exposure of the MIM component was necessary to generate a high joint strength. Likewise, oxidation on the metallic surface does not seem to have a significant impact on the joint strength. It was also found that samples with a greater surface roughness tend to show a more pronounced joining zone. This can be attributed to the fact that with a coarser blasting medium deeper "valleys" can be generated in the substrate or on the MIM component. As a consequence, more powder is deposited in these "valleys".

Joints of the same type seem to be fatigue resistant even under high loads. Heat treatment may not always be necessary. In functional areas such as threads, however, heat treatment is essential to ensure permanently high and homogeneous strengths to fulfil the function of a multifunctional and lightweight component.

\section{Conclusion and outlook}

The investigations demonstrated the high joint strength under both static and dynamic loads between MIM and additively manufactured components. It was found that the weakest part is the MIM component. Interactions in the interface, such as manual powder deposition, oxide layer formation and temperature differences are therefore negligible. An increase of the tensile strength could be achieved by hot isostatic pressing. To further increase the tensile strength, a heat treatment should follow and improve the MIM process.

By fusing the two technologies in hydraulic components, for example, new potentials and fields of application for more powerful aerospace components are opened up, which are also characterised by high resource efficiency in production.

\section{Acknowledgements}

This work was carried out within the framework of the German Aerospace Center (DLR) project Hydraulische System Innovation (Hyd-I), LuFo V-2, FKZ: 20Y1506E on behalf of the Federal Ministry for Economic Affairs and Energy (BMWi). The financial support is gratefully acknowledged.

\section{References}

[1] Vaithilingam, J. et al.: The effect of laser remelting on the surface chemistry of Ti6Al4V components fabricated by selective laser melting, Journal of Materials Processing Technology, 232, (2016), pp 1-8.

[2] Song, B. et al.: Effects of processing parameters on microstructure and mechanical property of selective laser melted Ti6Al4V, Materials and Design, 35, (2011), pp 120-125.

[3] Leuders, S. et al.: On the mechanical behaviour of titanium alloy TiAl6V4 manufactured by selective laser melting: Fatigue resistance and crack growth performance, International Journal of Fatigue, 48, (2012), pp. 300-307.

[4] Ferri; O. M.; Ebel, T.; Borman, R.: High cycle fatigue behaviour of Ti-6Al-4V fabricated by metal injection moulding technology. Materials Science and Engineering A, 504, (2009), pp. 107-113.

[5] Fores, F. H.: POWDER METALLURGY ABROAD. ADVANCES IN TITANIUM METAL INJECTION MOLDING. Powder Metallurgy and Metal Ceramics, 46, (2007), pp. 303-310.

[6] Ahuja, B. et al.: High Power Laser Beam Melting of Ti-6Al-4V on Formed Sheet Metal to achieve Hybrid structures. Laser 3D Manufacturing II, (2015).

[7] Schaub, A. et al.: Fabrication and Characterization of Laser Beam Melted Ti-6Al-4V Geometries on Sheet Metal. Fraunhofer Direct Digital Manufacturing Conference, (2014). 
[8] Schaub, A.: Grundlagenwissenschaftliche Untersuchung der kombinierten Prozesskette aus Umformen und Additive Fertigung. Dissertation, Lehrstuhl für Fertigungstechnologie, FriedrichAlexander-Universität Erlangen-Nürnberg, 2019.

[9] Amtsblatt der europäischen Union: Delegierte Verordnung (EU) 2019/1603 der Kommission. July 2019. 